\title{
Simulation of the Electron Tunneling Paths in Networks of Nano-particle Films
}

\author{
Milovan Šuvakov and Bosiljka Tadić \\ Department for Theoretical Physics, Jožef Stefan Institute, Box 3000, 1001 Ljubljana, \\ Slovenia \\ Milovan.Suvakov@ijs.si, Bosiljka.Tadic@ijs.si \\ http://www-f1.ijs.si/ tadic/, http://www-f1.ijs.si/ suvakov/
}

\begin{abstract}
Thin films of nano-particles deposited on substrates are important for new technological applications. Their physical properties depend crucially on particle size and structural inhomogeneity of the deposition. To systematically investigate these dependencies, we apply graph theory and simulations of voltage-driven tunneling conduction. We implement a network model of nano-particle films, based on data of particle positions on a two-dimensional plane. Assuming that connected particles on the network are within the tunneling distance, we then implement a continuous-time electron tunneling on the network and show how the current-voltage characteristics depend on the graph structure. We visualize the conduction paths through the network which correspond to the measured currents both in random and spatially inhomogeneous films.
\end{abstract}

Keywords: nano-particles; network; electron Tunneling Paths.

\section{Introduction}

In search of new materials for advanced technology applications [1] metallic nano-particles deposited on a two-dimensional substrate either as regular arrays [234] or as thin films [5] are being extensively investigated. Often thin films are grown by spin casting or methods that involve non-linear processes in which nano-particles move in a liquid, both leading to characteristically inhomogeneous structures, that can be varied via control parameters of the process 6 678. It has been recognized that the physical properties of these nano-particle arrays and thin films depend on the mutual positions of the nano-particles and global characteristics of the structure [9]. In particular, enhanced non-linear conduction properties have been observed in certain structures of nano-particles 101112131415 .

In order to systematically investigate the topology effects on the conduction in two-dimensional nano-particle structures, here we propose a numerical approach based on the graph theory and simulations of the electron tunneling processes. Our approach consists of two steps: 
- Mapping of an arbitrary nano-particle array to a graph;

- Implementation of electron-tunneling through the graph;

In the first part, as described below, the positions of nano-particles on a twodimensional substrate are exactly observed. The mapping of the structure to the graph enables quantitative study of the structure in many details. In the second part, the dynamics of voltage-driven electron tunnelings are strictly linked to the graph structure and thus their dependence on the structural elements of the graph can be easily traced. We demonstrate by simulations and by graphical means (visualizing the conduction paths) how the current flows through two such graphs, which correspond to two types of films made by random and by correlated deposition of particles.

\section{Mapping of Nano-particle Films to Networks}

\subsection{Particle Deposition}

For implementation of our numerical methods we assume that the positions of nano-particles on the substrate are known, e.g., from STM measurements or by statistical estimates based on the parameters of the deposition process. To illustrate our approach, we generate two types of films, shown in Fig. 1, by sequential deposition of particles of a unique size (determined by the grid spacing). In Fig 1(a) the deposition site for a new particle is randomly selected away from already occupied sites, resulting in a random homogeneous structure.

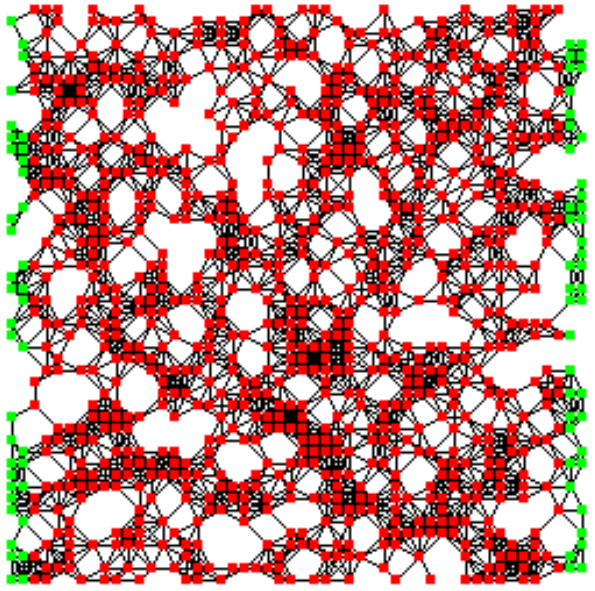

(a)

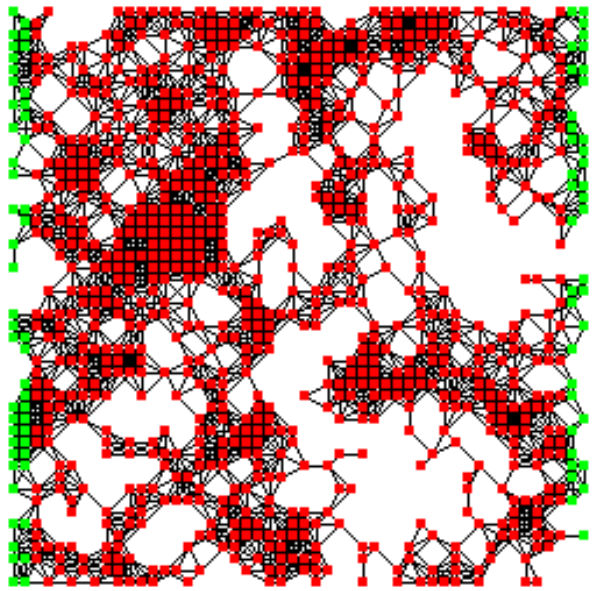

(b)

Fig. 1. Examples of the nano-particle films with (a) random and (b) correlated deposition at density $\rho=10 \%$ grid points covered by particles. Network edges are shown corresponding to the radius $r$ of 2 grid points, discussed in the text. 
In contrast to the random deposition, in Fig. 1(b) we apply self-avoiding randomwalk dynamics to select the deposition sites. In this case, the non-linear selfavoiding process leads to an inhomogeneous deposition, with inhomogeneity at different scales [16]. In both cases we put $N=1000$ particles on the rectangular plane with $100 \times 100$ grid points (see Fig. 1).

\subsection{Emergent Networks}

Next we make a nano-particle film network by connecting pairs of nano-particles, which are spaced within a small radius $r$ from each other. For the purposes of the tunneling processes that we would like to study on these films, the radius $r$ will be selected to coincide with the "tunneling distance", that is known for each nano-particle type. In our numerical experiment, we may vary $r$ from one to few grid points. The emergent networks have a variable, but limited node connectivity, which reflects the local inhomogeneity of the deposition. For instance, the networks shown in Fig. 1 have the average local connectivity $\langle k\rangle=9$ but with small dispersion $\sigma_{k 1}=2.62$ in the random deposition network, whereas $\sigma_{k 2}=4.05$ in the case of correlated deposition network. Further topological properties of such networks can be studied using the appropriate algorithms and the graph theory 1718 .

In the next section we implement the conduction via microscopic tunneling of charges through these networks. For this purpose it is important to check that for a given average particle density $\rho$ (surface coverage) and fixed radius $r$, the system is above the geometrical percolation point.

\section{Conduction}

For the simulations of the tunneling currents driven by voltage differences between two electrodes, the network structure (Fig. 1) is fixed by its adjacency matrix $\hat{\mathbf{A}}$. We then place the electrodes at left-most and right-most nodes of the network. By increasing the voltage difference, the conditions for the electron tunneling into the system of nano-particles from the side of higher voltage electrode are created. Increasing the voltage in small steps, the front of tunneling charges moves towards the low-voltage electrode and eventually touches the electrode when the voltage exceeds a threshold value $V_{T}$, which depends on the distance between electrodes and other details of the process, described below.

\subsection{Tunneling Implementation}

The energy of charged particles on a network of size $N$ is given by the Hamiltonian [10,12 13,19]:

$$
E=\frac{1}{2} \mathbf{Q}^{\dagger} M^{-1} \mathbf{Q}+\mathbf{Q} \cdot V^{e x t}+Q_{\mu} \Phi^{\mu}=E^{(1)}+E^{(2)}+Q_{\mu} \Phi^{\mu}
$$

with the vector of charges at nodes (nano-particles) $\mathbf{Q} \equiv\left\{Q_{i}\right\}, i=1,2 \cdots N$, the matrix of capacitancies $\hat{\mathbf{M}}$, and the potential of the electrodes $\Phi^{\mu}, \mu \in$ 
$\{+,-$,gate $\}$. The external potential is $V^{e x t}=M^{-1} \mathbf{C}_{\mu} \Phi^{\mu}$, where $\mathbf{C}_{\mu}$ is the vector of capacitance between dots and electrodes $\mu$. The microscopic structure of the underlying nano-particle array appears through the off-diagonal elements of the matrix $\hat{\mathbf{M}}$, i.e., via the adjacency matrix, i.e., $M_{i j}=-A_{i j}$ for $i \neq j$.

The inter-particle charge transport is a stochastic process in which tunneling of an electron between nodes $i \rightarrow j$ at time $t$ is governed by the probability distribution $p_{i j}(t)=\Gamma_{i j} \exp \left(-\Gamma_{i j} t\right)$, with the tunneling rate [1110.

$$
\Gamma_{i j}=-\frac{\Delta E_{i j} / e^{2} R}{1-\exp \left(\Delta E_{i j} / k_{B} T\right)},
$$

which is determined by the energy change $\Delta E_{i j}$ associated with the tunneling process. Here $e$ is the electron charge, $R$ is quantum resistance and $T$ is temperature.

Next we calculate the energy changes associated to the tunneling along the links of the network. After a single electron tunneling process from dot $a$ to dot $b\left(Q_{i}^{\prime}=Q_{i}+\delta_{b i}-\delta_{a i}\right)$ the change in the interaction energy term in Eq.(1), $\Delta E^{(1)}(a \rightarrow b)=E^{(1)^{\prime}}-E^{(1)}$ can be written as:

$$
\Delta E^{(1)}(a \rightarrow b)=\frac{1}{2} \sum_{i j}\left(Q_{i}+\delta_{b i}-\delta_{a i}\right) M_{i j}^{-1}\left(Q_{j}+\delta_{b j}-\delta_{a j}\right)-\frac{1}{2} \sum_{i j} Q_{i} M_{i j}^{-1} Q_{j}
$$

Using $M_{i j}^{-1}=M_{j i}^{-1}(3)$ becomes:

$$
\Delta E^{(1)}(a \rightarrow b)=\sum_{i} Q_{i}\left(M_{i b}^{-1}-M_{i a}^{-1}\right)+\frac{1}{2}\left(M_{a a}^{-1}+M_{b b}^{-1}-M_{a b}^{-1}-M_{b a}^{-1}\right) .
$$

Similarly, the change in the second energy term of Eq.(1), which is associated with the interaction with the external potential, $\Delta E^{(2)}(a \rightarrow b)=E^{(2)^{\prime}}-E^{(2)}$ becomes

$$
\Delta E^{(2)}(a \rightarrow b)=\sum_{i}\left(Q_{i}+\delta_{b i}-\delta_{a i}\right) V_{i}^{e x t}-\sum_{i} Q_{i} V_{i}^{e x t}=V_{b}^{e x t}-V_{a}^{e x t} .
$$

In addition, the contribution from the tunneling processes between electrodes and dots is computed. A tunneling process between an electrode and a dot $a$ $\left(Q_{i}^{\prime}=Q_{i} \pm \delta_{a i}\right)$ contributes the energy changes in both terms, which are:

$$
\begin{aligned}
\Delta E^{(1)}(a \leftrightarrow \pm) & =\frac{1}{2} \sum_{i j}\left(Q_{i} \pm \delta_{a i}\right) M_{i j}^{-1}\left(Q_{j} \pm \delta_{a j}\right)-\frac{1}{2} \sum_{i j} Q_{i} M_{i j}^{-1} Q_{j} \\
& = \pm \sum_{i} Q_{i} M_{i a}^{-1}+\frac{1}{2} M_{a a}^{-1},
\end{aligned}
$$

and

$$
\Delta E^{(2)}(a \leftrightarrow \pm)=\sum_{i}\left(Q_{i} \pm \delta_{a i}\right) V_{i}^{e x t}-\sum_{i} Q_{i} V_{i}^{e x t}= \pm V_{a}^{e x t}
$$


We can write the expressions (4) and (6) can be written in a concise form using the appropriate variables $V_{c} \equiv \sum_{i} Q_{i} M_{i c}^{-1}$. We obtain:

$$
\begin{gathered}
\Delta E^{(1)}(a \rightarrow b)=V_{b}-V_{a}+\frac{1}{2}\left(M_{a a}^{-1}+M_{b b}^{-1}-M_{a b}^{-1}-M_{b a}^{-1}\right), \\
\Delta E^{(1)}(a \leftrightarrow \pm)= \pm V_{a}+\frac{1}{2} M_{a a}^{-1} .
\end{gathered}
$$

In the case when $C<<C_{q}$ the off-diagonal elements of inverse capacitance matrix fall off exponentially and in calculations of $V_{c}$ we can use only nearst neighbor terms, which speeds up the calculations.

In the simulations, following an increase of the voltage on one electrode, it takes some transient period until the distribution of charges become stationary. We then sample the charges at all nodes and currents through all links of the network. The relevant quantities are measured in the following units: charge $Q[e]$, voltage $V\left[e / C_{g}\right]$, time $t\left[R C_{g}\right]$ and current $I\left[e / R C_{g}\right]$. In this paper we focus on certain properties of current in the limit $C \ll C_{g}$ and we set $T=0$, where the tunneling effects are most pronounced, and $\Phi^{\text {gate }}=\Phi^{-}=0$.

The numerical implementation in $\mathrm{C}++$ is done according to the following steps:

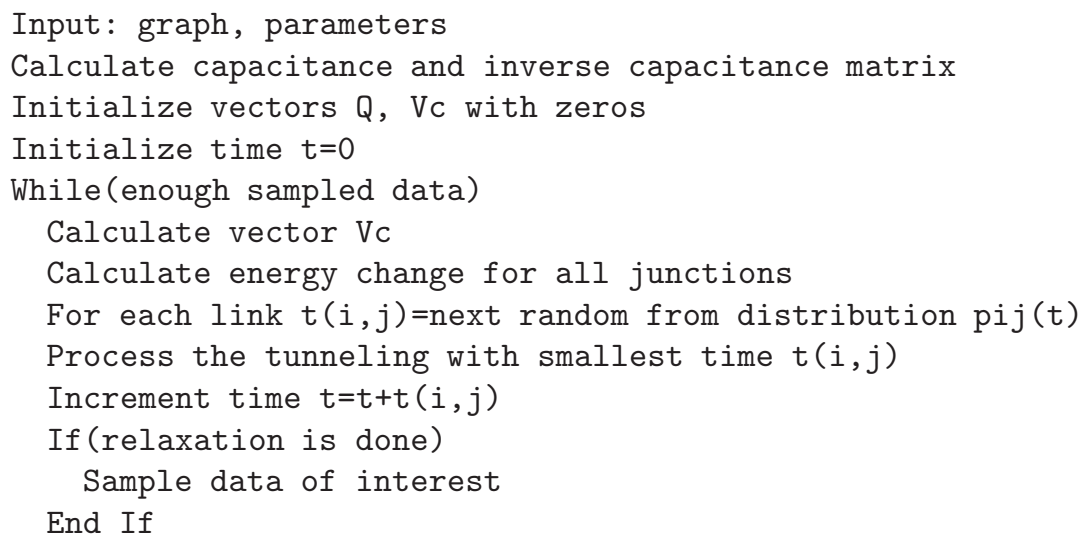

\subsection{Conduction Paths and Nonlinear Currents}

As mentioned above, driven by the external potential, the charges are entering the system from the higher-voltage electrode. When the voltage is small, the charges can screen the external potential, in which case the system may become static and there is no current. For the voltage larger than a threshold value $V_{T}$ the screening does not occur and, after some transient time, a stationary distribution of charges over dots sets-in with a constant current through the system. In general, the experimental measurements of the current-voltage characteristics in nano-particle arrays revealed a non-linear dependence [13] according to the law

$$
I \sim\left(V / V_{T}-1\right)^{\zeta}
$$




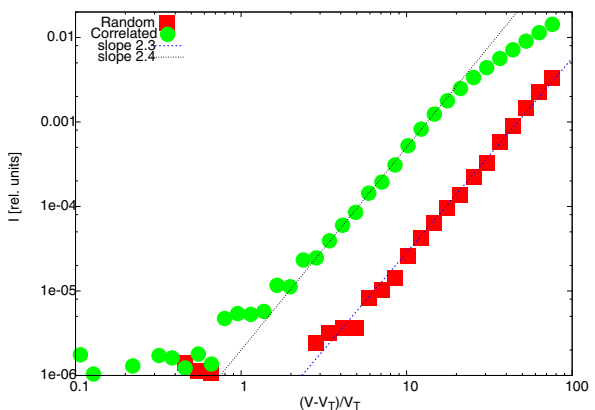

(a)

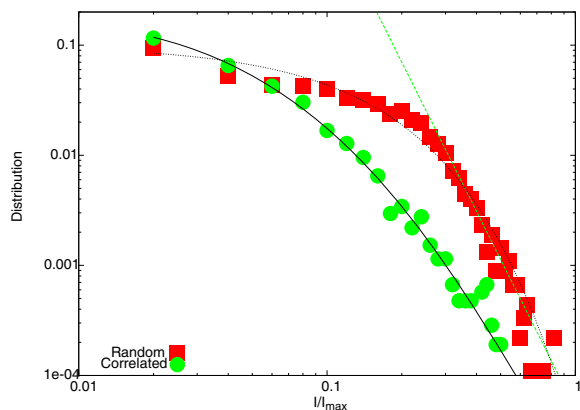

(b)

Fig. 2. Conduction on the networks shown in Fig. 1: (a) Non-linear I-V curves with solid lines representing the power-law fits, according to Eq. (10); (b) Distribution of normalized currents over links. The solid lines represent fits with Eq. (11).

in a range of values of voltage above $V>V_{T}$. In Fig. 2(a) we show the results of the simulated current-voltage curves in our two networks. They exhibit a typical non-linear behavior with the exponent $\zeta>2$. The difference between these two curves appears in the saturation region, visible in the case of inhomogeneous network for voltage around $30 V_{T}$. This saturation can be explained by the existence of a large number of bottle necks in the inhomogeneous network structure.

For the fixed value of voltage $V=10 V_{T}$ we demonstrate how the electrons flow through the network by monitoring the currents through each link. All data are collected after a transient (relaxation) period. The distributions of currents through the links are shown in Fig. 2(b) for the two network topologies. In the case of random network there is a well defined power-law tail with a large slope of $\approx 4.5$ (solid line). For the correlated network we found that the flow distribution can be well fitted with a q-exponential form 20.

$$
P(x)=B_{0}\left(1-(1-q) \frac{I / I_{\max }}{x_{0}}\right)^{1 /(1-q)},
$$

often found in non ergodic dynamical systems. Here we find $B_{0}=0.22, q=1.21$ and $x_{0}=0.03$. Applying, for a comparison, the same expression to fit the flow data collected on the random structure, (doted line Fig. 2(b)), we find quite different values: $B_{0}=0.1, q=1.01$ and $x_{0}=0.12$. These values are compatible with the even distribution of flow over links in the random deposition structure, and the absence of links which play a special role in the conduction process.

The observed quantitative difference in the current flow over these two networks are graphically illustrated by visualization of the conduction paths with an appropriate java applet. The images are shown in Fig. 3. Different levels of gray color are proportional to the current flow along the network links. 


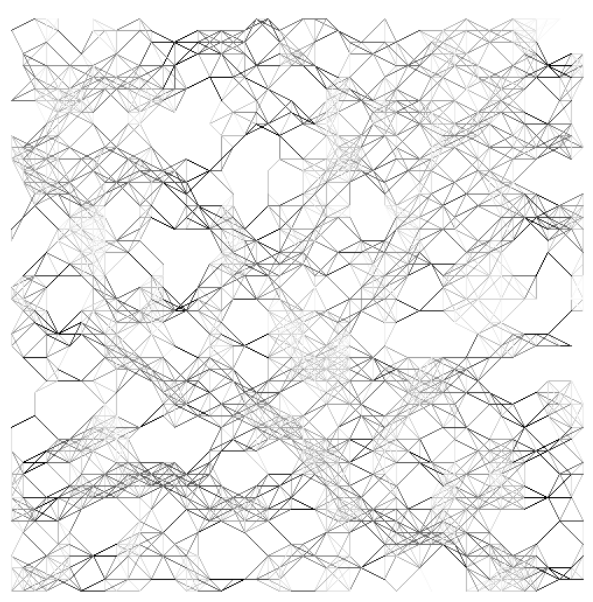

(a)

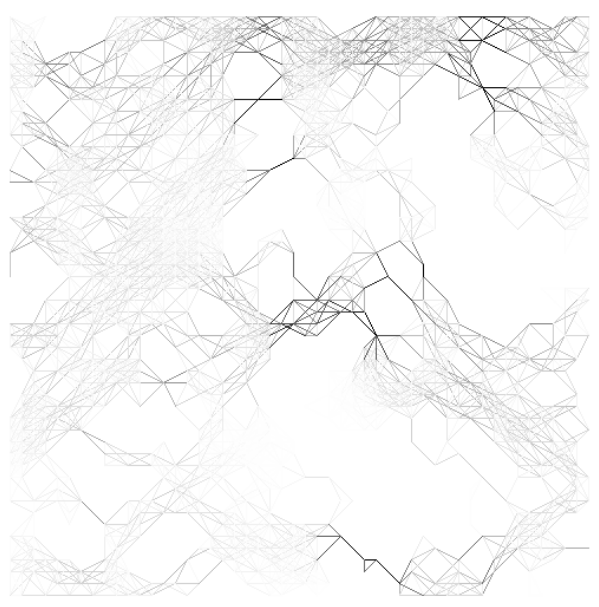

(b)

Fig. 3. Conduction paths on networks shown in Fig. 1: (a) random, (b) correlated structure. Intensity in the gray scale is proportional to the flow over links.

\section{Conclusions}

We have implemented a numerical algorithm for analysis of the electron tunneling conduction through nano-particle arrays on a two-dimensional substrates. The inputs in the algorithm are positions of the nano-particles in the x-y plane, that can be obtained either from the appropriate measurements in the real systems or from a theoretical model.

We have demonstrated how the algorithm works in two types of networks, corresponding to a random and a correlated deposition of nano-particles. In particular, the flow over the links has been studied quantitatively by the statistical distributions and visualized graphically. In both networks we obtain a non-linear current-voltage characteristics with most available experimental data of nano-particle arrays. Furthermore, we have demonstrated that both of these measures appear to be dependent on the actual structure of the network. Hence, these measures of the current flow can be used as a tool to characterize the film structure-conduction properties.

The graphic visualization algorithm developed in this work (made available for an interactive application on the author's Web site) can be used for visualization of flow in a general case of planar graphs.

\section{Acknowledgments}

We thank the financial support by the Marie Curie Research and Training Network MRTN-CT-2004-005728 project and by the programme P1-0044 of the Ministry of high education, science and technology (Slovenia). 


\section{References}

1. T. Bigioni, X. Lin, T. Nguyen, E. Corwin, T. Witten, and H. Jeager. Kinetically driven self assembly of highly ordered nanoparticle monolayers. Nature Materials, 5:265, 2006.

2. C. I. Duruöz, R. M. Clarke, C. M. Marcus, and J. S. Harris, Jr. Conduction threshold, switching, and hysteresis in quantum dot arrays. Phys. Rev. Lett., 74(16): 3237-3240, 1995.

3. Ç. Kurdak, A. J. Rimberg, T. R. Ho, and J. Clarke. Activated transport and scaling behavior in the current-voltage characteristics and coulomb-blockade oscillations of two-dimensional arrays of metallic islands. Phys. Rev. B, 57(12):R6842-R6845, Mar 1998.

4. A. J. Rimberg, T. R. Ho, and J. Clarke. Scaling behavior in the current-voltage characteristic of one- and two-dimensional arrays of small metallic islands. Phys. Rev. Lett., 74(23):4714-4717, Jun 1995.

5. P. Moriarty. Nanostructured materials. Reports of Progress in Physics, 64:297-381, 2001.

6. P. Moriarty, M. D. R. Taylor, and M. Brust. Nanostructured cellular networks. Phys. Rev. Lett., 89(24):248303, 2002.

7. Z. Konstantinovic, M. G. del Muro, M. Varela, X. Batlle, and A. Labarta. Particle growing mechanisms in ag-zro2 and au-zro2 granular films obtained by pulsed laser deposition. Nanotechnology, 17:4106, 2006.

8. M. Brust, D. Bethell, Kiely C. J., and D. J. Schiffrin. Self-assembled gold nanoparticle thin films with nonmetallic optical and electronic properties. Langmuir, 14(19):5425 -5429, 1998.

9. C. P. Martin, M. O. Blunt, and P. Moriarty. Nanoparticle networks on silicon: Self-organized or disorganized? Nano Lett., 4(12):2389 -2392, 2004.

10. B. K. Ferry and S. M. Goodnick. Transport in Nanostructures. Cambridge University Press, 1997.

11. U. Geigenmüller and G. Schön. Single-electron effects in arrays of normal tunnel junctions. Europhysics Letters, 10:765-+, December 1989.

12. N. S. Bakhvalov, G. S. Kazacha, K. K. Likharev, and S. I. Serdyukova. Statics and dynamics of single-electron solitons in two-dimensional arrays of ultrasmall tunnel junctions. Physica B Condensed Matter, 173:319-328, September 1991.

13. A. A. Middleton and N. S. Wingreen. Collective transport in arrays of small metallic dots. Phys. Rev. Lett., 71(19):3198-3201, 1993.

14. M. N. Wybourne, L. Clarke, M. Yan, S. X. Cai, L. O. Brown, J. Hutchison, and J. F. W. Keana. Coulomb-blockade dominated transport in patterned gold-cluster structures. Jpn. J. Appl. Phys., 36:7796-7800, 1997.

15. R. Parthasarathy, X. Lin, K. Elteto, T. F. Rosenbaum, and H. M. Jeager. Percolating through networks of random thresholds: Finite temperature electron tunneling in metal nanocrystal arrays. Phys. Rev. Lett., 92:076801, 2004.

16. G. F. Lawler. Intersections of Random Walks. Birkhauser-Boston (1996).

17. R. K. Ahuja, T. L. Magnanti, and J. B. Orlin. Network Flows: Theory, Algorithms, and Applications. Prentice Hall, 1993.

18. M. Šuvakov and Tadić B. Topology of cell-aggregated planar graphs. Springer (Berlin) Part III, 3993:1098, 2006.

19. M. Šuvakov and B. Tadić. Transport processes on homogeneous planar graphs with scale-free loops. Physica A, 372:354-361, 2006.

20. Tsallis C. Possible generalization of boltzmann-gibbs entropy. J. Stat. Phys, 52:479, 1988. 